Australia, accounting for nearly half the overdose fatalities in the country.

\section{CONCLUSION}

The frequency of heroin use among IDUs has risen substantially over the last few years, and all indicators of heroin use suggest that there has been an increase in the number of heroin users in NSW. Harm to public health associated with injecting heroin use continues to be prominent, particularly overdose fatalities.

\section{REFERENCES}

1. Wardlaw, G. Illicit Drug Reporting System. Consultant's Report to the Commonwealth. Canberra: Department of Human Services and Health and Wardlaw Consulting Pty Ltd, 1994.

2. Australian Institute of Health and Welfare. 1998 National Drug Strategy Household Survey: First Results. Canberra: Australian Institute of Health and Welfare, 1999. it

The following IDRS reports are available through the National Drug and Alcohol Research Centre by telephone on (02) 9398 9333; by fax on (02) 93997143 :

- Drug Trends Bulletin: a free quarterly report from the IDRS that contains an update on recent drug trend information;

- State reports: annual reports that contain the findings of the IDRS from each state and territory $(\$ 10$ each);

- National report: an overview of Australian drug trends $(\$ 10)$.

For a summary of the latest drug trends visit the IDRS Web site at: www.med.unsw.edu.au/ndarc/idrs.

\title{
NSW DRUG SUMMIT UPDATE
}

Kee Foong and Jennifer Gray

NSW Drug Programs Bureau

NSW Department of Health

In May 1999, the NSW Drug Summit was held at the NSW Parliament. For one week members of State Parliament, drug experts, parents, former drug users, and community leaders, met to decide how best to deal with the drug problem in NSW. The summit endorsed a number of resolutions, the majority of which were accepted by the NSW Government. A Government Plan of Action (GPA) was developed, and this article describes initiatives developed to implement this plan.

The GPA describes a comprehensive, 'whole of government' and 'whole of community' approach to the prevention and management of illicit drug misuse. Over $\$ 176$ million has been provided by the State Government to implement a range of new initiatives over the next four years. This has been the most significant enhancement in funding to drug and alcohol services since the National Drug Strategy in 1985. To coordinate this significant investment the State Government has established the Office of Drug Policy within the NSW Cabinet Office. An expert advisory committee, reporting to the Premier, has also been established and is chaired by Professor Ian Webster.

\section{IMPLEMENTATION}

NSW Health has received significant enhancements. The new monies have been allocated to significantly enhance service provision; and to ensure that a system built on quality, equity and fairer access is in place. The Drug Programs Bureau (formerly the Drug and Alcohol Directorate) within the Public Health Division, and the Area Health Services (AHS), have primary responsibility for more than 100 projects resulting from the GPA. 


\section{A strategic approach to delivering drug treatment services}

The Summit recognised the importance of clear strategies, as well as the need for quality services. A NSW Drug Treatment Services Plan (DTSP) has been developed, which describes the priorities for treatment over the next five years, and how the Government will achieve an appropriate level and mix of treatment options. The DTSP ensures that NSW has in place an effective and responsive system of services for people affected by drug use.

\section{Area Health Service Project Plans}

As a first step to allocating funding and implementing the DTSP, each AHS was required to submit a project plan to the NSW Department of Health. This approach allows for a coordinated response across the state, but tailored to individual Area needs and priorities. Comprehensive AHS plans now exist for a range of projects, including: home detoxification, data collection, methadone maintenance treatment, and additional specialist staffing positions such as nurses and counsellors.

\section{New and Expanded Drug Treatment Services}

New or enhanced services will be established in a number of areas identified as having a high need. These areas include detoxification facilities in Northern Rivers, Wentworth and Central Coast Area Health Services; multipurpose drug and alcohol units in New England, Mid-North Coast and South Western Sydney Area Health Services; and increased residential beds for non-government organisations providing rehabilitation services.

Ambulatory and home detoxification services will be expanded, which will enhance accessibility to detoxification services, ease pressure on hospital beds, and extend the range of treatment services available to the community. Ambulatory and home detoxification is being trialled in the Central Sydney, South Western Sydney, Southern, Greater Murray and the Far West Area Health Services. Greater general practitioner (GP) participation in the program is being encouraged.

\section{Expansion of pharmacotherapies and comprehensive case management}

Access to pharmacotherapy treatment programs will be increased across all AHSs. Health and social outcomes will be improved by:

- increasing the number of places available in the public methadone maintenance treatment program;

- expanding counselling and case management services in the public methadone program as well as those managed by GPs and pharmacists.

Treatment agreements are to be put in place across the pharmacotherapy treatment programs. These agreements will provide a mechanism for improving communication between the patient and the service provider, and will improve the quality of treatment in NSW by outlining the responsibilities of both the client and the provider.

\section{Improved data and information}

The collection, collation and availability of accurate and consistent data is essential for effective service planning. For the first time, all AHSs will develop a standardised data collection and reporting mechanism that will function across all drug treatment services funded by NSW Health. Data will be kept describing client profiles and service activity and funds have already been allocated to all Areas for this project.

\section{Improved ability of primary care providers to provide drug and alcohol services}

Area Health Services will be working closely with their local Divisions of General Practice to increase the capacity of GPs to provide drug and alcohol services. Eleven AHSs have initially been provided with funding for 1999-2000 and 2000-2001. Funding will be extended to all Areas from 2001-2002 onwards. Project officers will undertake activities to improve links between GPs and drug and alcohol services, and to develop GP training initiatives.

A strategy is also being developed to increase the capacity of nurses in general health care settings to handle patients with drug and alcohol issues. Clinical practice guidelines will complement the strategy and both will soon be released.

It can be seen that, almost one year after the Drug Summit, much has been achieved. The breadth of projects covered should lead to:

- better availability of information

- a greater number and range of services

- better service planning

- a highly skilled workforce

- quality drug and alcohol services accessible to those in need.

It is a large task but NSW Health is committed to implementing the GPA to reduce the problems resulting from drug use, and to provide services to those who require assistance. it 